\title{
Community
}

\section{Community News}

DOI 10.1515/aot-2015-0009

\section{International Year of Light started}

The International Year of Light (ILY) and Light-based Technologies 2015, as it is named correctly, is 'a global initiative that will highlight to the citizens of the world the importance of light and optical technologies in their lives, for their future, and for the development of society' as the Optical Society of America OSA puts it. The resolution proposing an IYL 2015 was placed before the 190th Session of the UNESCO executive board. It was adopted by the United Nations during a plenary meeting of the 68th Session of the UN General Assembly with the intention 'to raise awareness of how optical technologies promote sustainable development and provide solutions to worldwide challenges in energy, education, agriculture, communications and health'.

\section{Embracing Muslim heritage: IYL partners with 1001 inventions}

The IYL 2015 launches at the UNESCO headquarters in Paris on 19 January 2015. But this is just the official start of a large variety of events. One particularly interesting activity is brought in by IYL funding partner - 1001 Inventions. British-based 1001 Inventions has the specific mission to raise awareness of the contributions to science, technology and culture from the Golden Age of Muslim Civilization, and will play a key role during IYL 2015 to promote and celebrate the 10th century pioneer Ibn Al-Haytham. Ibn Al-Haytham's seminal work on optics Kitab al-Manazir (The Book of Optics) was written around 1015 , and its 1000th anniversary is listed explicitly in the United Nations resolution on IYL 2015 as a focal point of celebration.

There are more major scientific anniversaries that will be celebrated during IYL 2015 (as listed by the comprehensive IYL site at Wikipedia):

- 1015: Works on optics by Ibn Al-Haytham

- 1815: The notion of light as a wave proposed by Fresnel

www.degruyter.com/aot

(c) 2015 THOSS Media and De Gruyter
- 1865: The electromagnetic theory of light propagation proposed by Maxwell

- 1915: Einstein's theory of the photoelectric effect in 1905 and of the embedding of light in cosmology through general relativity

- 1965: Discovery of the cosmic microwave background by Penzias and Wilson, and Charles Kao's achievements concerning the transmission of light in fibers for optical communication

\section{IYL activities around the world}

Photonics technologies has received growing attention in recent years. Light is seen as a crucial ingredient for the development of life and now people see it is a crucial means to further improve living conditions for the majority of people. Accordingly, the idea was embraced by many people and organizations around the world. Most prominently among them are the academic and industrial representatives of photonic research and industry. Activities can be found on almost all continents.

While UNESCO is designated as the lead agency, they cooperate with a consortium of hundreds of worldwide partner countries, accompanied by the UNESCO International Basic Sciences Programme (IBSP). Most physical and optics societies are involved, such as the African Physical Society AfPS, European Physical and Optical Societies EPS and EOS, the German Physical Society (DPG), the Chinese Optical and Physical Societies COS and CPS, the American Physical Society APS, the Optical Society of America OSA, International Society for Optics and Photonics SPIE and many more.

More information can be found on the respective websites, a small selection are cited here:

- International IYL page: www.light2015.org

- EPS - page with news, blogs and events in Europe www.epsnews.eu/category/iyl-2015/

- OSA IYL page: www.osa.org/en-us/about_osa/ international_year_of_light/

- German IYL page (event calendar) www.jahr-deslichts.de/. 


\section{$€ 100,000$ awarded for terabit technology}

Professor Christian Koos of the Karlsruhe Institute of Technology (KIT) is awarded this year's Prize for Applied Research from the federal state of BadenWürttemberg. Koos conducts research on nanophotonic components and novel techniques of optical high-speed communication. Together with colleagues at the École Polytechnique Fédérale de Lausanne (EPFL) he and his team succeeded in transmitting a data stream of 1.44 terabit per second using a miniaturized frequency comb as an optical source.

Christian Koos and his co-workers conduct research on new optical components and transmission techniques for terabit communications at the KIT's Institute of Photonics and Quantum Electronics and at its Institute of Microstructure Technology. This work allows to miniaturize optical components and enables integration on optical microchips. 'We seek to reduce to the size of current rack-filling transmitter and receiver systems to a matchbox format. This makes systems not only less expensive, but also saves electricity; our components are among the most energy-efficient worldwide', explains Koos. Besides terabit communication in large data centers, he envisages a number of other possible applications of his components, e.g., in optical sensing and metrology and in life sciences, where optical chips are also becoming more and more important.

Using optical frequency combs instead of conventional laser light is a key concept to speed up data transmission: Optical frequency combs consist of thousands of closely spaced spectral lines whose distances are defined very precisely. Each of these spectral lines can be used to transmit an optical data stream. 'A single frequency comb source can replace a large number of conventional lasers in a data transmission system', says Jörg Pfeifle, who conducts research on frequency combs in the group of Christian Koos. In combination with nano-scale components that can be integrated in larger quantities on one tiny silicon chip, optical frequency combs allow data streams to be processed with highest transmission rates in an energy-efficient way. Together with colleagues of the École Polytechnique Fédérale de Lausanne (EPFL), last year, Koo and his team were the first research group in the world to show that miniaturized optical frequency comb sources allow data transmission rates of up to 1.44 terabit per second over $300 \mathrm{~km}$.

Christian Koos intends to spend part of the prize money on investments into equipment opening up new possibilities in nanofabrication and data transmission. In addition, he also plans to make use of the prize money as 'scientific venture capital', allowing young scientists room for creative research. 'I would like to use these funds to finance work of post-graduates and post-docs who want to try out new ideas on their own', says Koos.

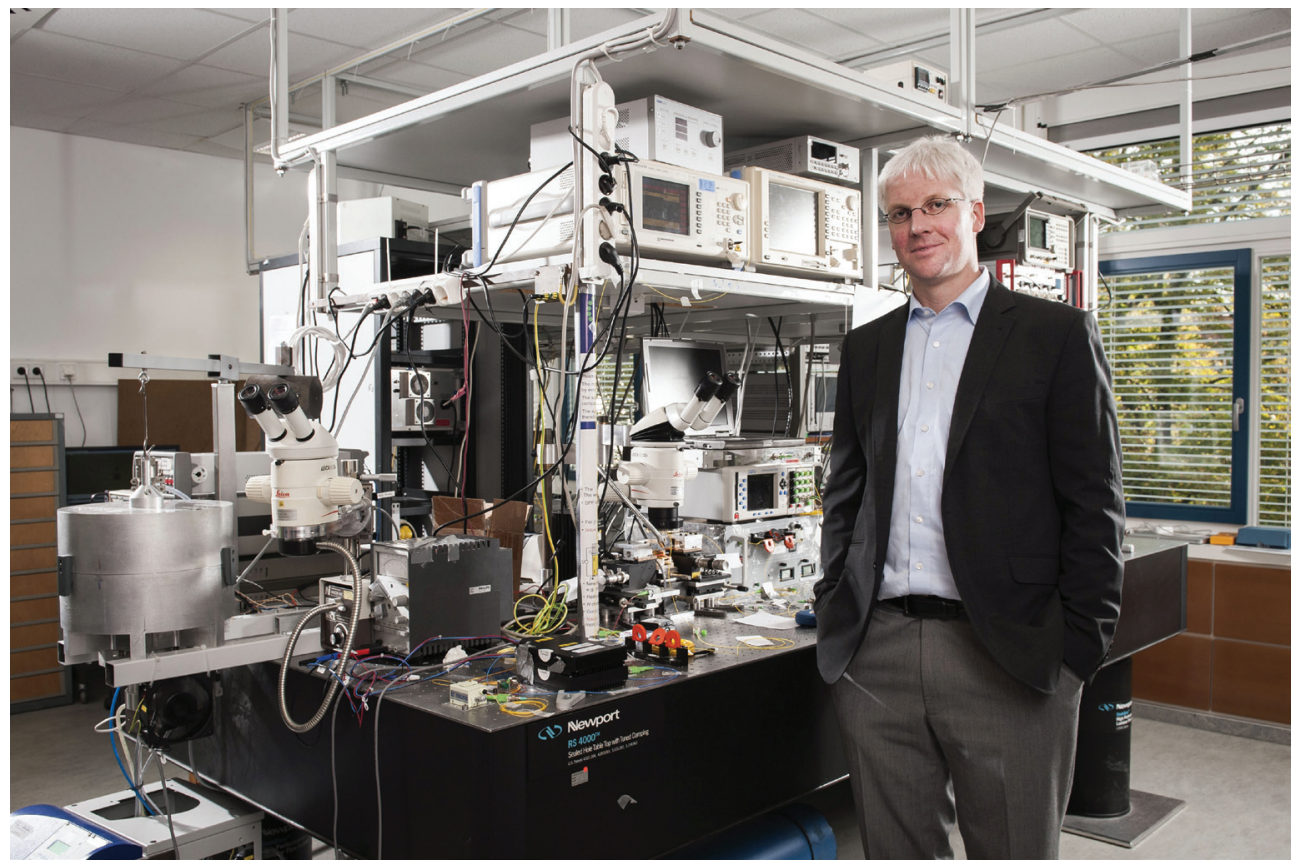

Christian Koos is awarded this year's Prize for Applied Research from Germany’s federal state of Baden-Württemberg (photo: Markus Breig, KIT). 
The Baden-Württemberg State Ministry for Science, Research, and Art awards the State Research Prize to outstanding scientists. This most highly endowed research prize of any German federal state in the amount of $€ 200,000$ is given in equal parts to one scientist each in basic research and applied research. The award ceremony was held in Stuttgart on December 8, 2014.

Christian Koos has been at the Karlsruhe Institute of Technology since 2010. Since 2013, he has headed the Institute of Photonics and Quantum Electronics and has been a member of the Management Board of the Institute of Microstructure Technology. The work awarded with the State Research Prize is part of the multi-disciplinary training program of the Helmholtz International Research School for Teratronics (HIRST), whose coordinator and spokesperson Christian Koos has been since 2012.

He studied electrical engineering and computer technology at the then University of Karlsruhe (TH), now the KIT, between 1997 and 2002. He finished his diploma thesis at Massachusetts Institute of Technology (MIT). Afterwards, he obtained his doctorate at the High-Frequency and Quantum Electronics Laboratory of the University of Karlsruhe (TH), where he also did research as a post-doc. After spending 2 years with Carl Zeiss AG, where he headed the technology radars for Nanotechnology and Metrology, Koos returned to Karlsruhe in 2010. Based on his research on nanophotonics and optical data transmission, the 36-year-old scientist was awarded a series of prizes and grants in recent years. In 2011, Koos obtained an 'ERC Starting Grant' in the amount of 1.5 million euro by the European Research Council. One year later, he won the Alfried Krupp Prize for Young University Teachers, which is endowed with 1 million euro. Christian Koos has authored and coauthored more than 200 publications in scientific journals and conference proceedings, more than 140 conference presentations, and more than 20 patent applications. And he will be guest editor of the Advanced Optical Technologies 2/2015 focus issue on 'Integrated Photonic Devices: Sensors, Materials, systems', together with Meint Smit (TU Endhoven, The Netherlands) and Peter O’Brien (Tyndall National Institute, Cork, Ireland). 